\title{
Changed functional connectivity at rest in functional illiterates after extensive literacy training
}

\author{
Bahram Mohammadi ${ }^{1,2 \dagger}$, Thomas F. Münte ${ }^{2,3 * \dagger}$ (D), David M. Cole ${ }^{4}$, Amir Sami ${ }^{1}$, Melanie Boltzmann ${ }^{5,6,7}$ and \\ Jascha Rüsseler ${ }^{5,6}$
}

\begin{abstract}
Background: About 6.2 million adults in Germany cannot read and write properly despite attending school for several years. They are considered to be functional illiterates (FI). Since the ability to read and write is crucial for being employed and socially accepted, we developed a special literacy training to overcome these deficits.

Methods: In this study, we investigate training-related changes in intrinsic functional connectivity (iFC) at rest in a group of $20 \mathrm{Fl}$ and 20 adult normal readers using resting state functional magnetic resonance imaging (rsfMRI). We used independent component analysis (ICA) to define different networks.

Results: Before training, the between group analysis showed increased iFC in FI in a left-fronto-parietal network (LFPN; anterior insula, medial frontal cortex, lateral and frontal parietal regions) and in the Basal Ganglia network (BGN: thalamus, caudate, putamen, pallidum, amygdala, supplementary motor cortex and cingulate gyrus).

Furthermore, the Visual Network-1 (VN1; temporal occipital fusiform gyrus, lateral occipital cortex, occipital pole, lingual gyrus, thalamus) showed decreased iFC in Fl. After training the Fl group showed reversal of the "hyperconnectivity" in middle frontal gyrus and in the frontal orbital cortex and between supramarginal gyrus and the BGN. Furthermore, functional connectivity increased in FI VN1 (lateral occipital cortex, insular cortex). These changes in connectivity correlated with gains in reading speed and spelling accuracy.

Conclusions: These findings show that poor reading and writing abilities are associated with abnormalities in iFC in several brain areas subserving cognitive processes important for reading. Intensive literacy training induces changes in the functional connectivity between and within neural networks important for literacy skills.
\end{abstract}

Keywords: Functional connectivity, Functional illiteracy, Independent component analysis, Training intervention

\section{Introduction}

While fluent reading is the default in developed countries and most children learn to read and write without major problems, reading and writing can be disturbed. Much research has been devoted to developmental dyslexia, an

\footnotetext{
* Correspondence: Thomas.muente@neuro.uni-luebeck.de

${ }^{+}$Bahram Mohammadi and Thomas F. Münte contributed equally to this work.

2Department of Neurology, University of Lübeck, Ratzeburger Allee 160, 23562 Lübeck, Germany

${ }^{3}$ Institute of Psychology II, University of Lübeck, Lübeck, Germany

Full list of author information is available at the end of the article
}

unexpected difficulty with accurate and fluent reading which occurs despite normal intelligence, motivation, and exposure to adequate reading instruction. Developmental dyslexia occurs in $5-17 \%$ of the children [68] and problems may persist into adulthood. Functionally illiterate (FI) adults show even greater difficulties during reading acquisition than dyslexic individuals. Although they received the same reading and writing instructions as typically developing readers, FI left school with literacy skills that are at least three to four years below the expected level of performance [23, 24, 30, 80]. Typically, FI are unable to read and understand even short sentences. Single

(c) The Author(s). 2020 Open Access This article is licensed under a Creative Commons Attribution 4.0 International License, which permits use, sharing, adaptation, distribution and reproduction in any medium or format, as long as you give appropriate credit to the original author(s) and the source, provide a link to the Creative Commons licence, and indicate if changes were made. The images or other third party material in this article are included in the article's Creative Commons licence, unless indicated otherwise in a credit line to the material. If material is not included in the article's Creative Commons licence and your intended use is not permitted by statutory regulation or exceeds the permitted use, you will need to obtain permission directly from the copyright holder. To view a copy of this licence, visit http://creativecommons.org/licenses/by/4.0/. 
word reading of frequently occurring words is sometimes uncompromised [24]. Recent surveys conclude that there are about 6.2 million FI in Germany (i.e. $12.1 \%$ of the adult population [34];), with similar prevalence rates in France (9\% [2];), and the UK (16\% [82];). Theories of functional illiteracy $[22,24]$ have focused on environmental factors in childhood concerning school (e.g. truancy, inappropriate instructions, repetition of classes) and the family environment (e.g. neglect, drug abuse of parents, abuse, numerous siblings etc.). Recent research suggests that these negative experiences do not apply for all individuals with low literacy skills, and are also not sufficient to let someone become functionally illiterate $[24,80]$. Accordingly, some researchers proposed that FI results from cognitive deficits coupled with environmental disadvantages [10, 24, 29, 31]. Moreover, a relationship between functional illiteracy and developmental dyslexia is assumed. As an example, Greenberg et al. [31] consider functional illiteracy as an adult form of developmental dyslexia not adequately treated in childhood (see also [80]). Accordingly, several studies showed similarities between the cognitive profiles and neurobiological underpinnings of reading in FI and in dyslexic readers [24, 29, 31, 33, 64].

The ability to read is supported by several distributed networks of different brain regions. Reviewing mainly task-based fMRI-studies, Grigorenko [32] identified three important brain circuits: A frontal system (inferior frontal gyrus), a temporo-parietal system (angular and supramarginal gyri, posterior part of the superior temporal gyrus [STG]), and an occipito-temporal system (occipito-temporal area and posterior parts of the middle and inferior temporal gyri). It has been shown that the frontal and the temporo-parietal systems are involved in phonological processing [32] and in grapheme-to-phoneme conversion. The occipito-temporal system is involved in "whole-word" recognition of word forms [69].

Resting-state fMRI (rsfMRI) can be used to study intrinsic functional connectivity (iFC) of the brain. It is detected by examining inter-regional correlations in spontaneous low-frequency fluctuations $(<0.1 \mathrm{~Hz})$ in the rsfMRI-signal [8]. Koyama et al. [47] used rsfMRI to investigate $\mathrm{iFC}$ of the reading networks at rest. The authors chose six seeds roughly corresponding to the three reading systems described previously by Grigorenko [32] and in meta-analyses by Turkeltaub et al. [79] and Bolger et al. [9]: left gyrus fusiformis, left superior temporal gyrus, left temporo-parietal junction, left precentral gyrus, left inferior frontal gyrus, and left inferior occipital gyrus. The observed connectivity patterns largely overlapped with findings from task-based fMRI-studies during word reading. Furthermore, negative iFC was reported for several regions involved in effortful and controlled processing (i.e. dorsolateral prefrontal and superior parietal areas) as well as in the default mode network. The posterior left inferior frontal gyrus (postLIFG) and the posterior left middle temporal gyrus (post-LMTG) were identified as possible loci of functional integration among the reading networks. These findings demonstrate the usefulness of rsfMRI to study the functional organization of reading in the brain. It is important to note, however, that many studies have demonstrated that reading engages many regions beyond the aforementioned reading networks and in particular limbic and subcortical regions [1, 3, 41, 53, 54].

A handful of studies have used rsfMRI to investigate the differences in iFC in reading networks of dyslexic and typically reading children [86] and/or explored changes in iFC of reading-related brain networks related to remediation $[42,43,48]$. Dyslexics differed from typical readers in iFC between the intraparietal sulcus and the visual word-form area as well as in the connections of these structures to the left-middle frontal gyrus [86]. Schurz et al. [66] observed reduced task-related and resting-state connectivity for dyslexics between seeds in left posterior temporal areas and the inferior frontal gyrus. This implies that the disruption of functional integration between frontal and temporal brain areas in dyslexic readers is a permanent one as it is present during task performance and in the absence of a task. Exploring the effects of reading remediation on iFC, Koyama et al. [48] studied (1) dyslexic children with current reading and spelling difficulties, (2) dyslexic children that received training and had only reading difficulties at test (partly remediated group), (3) fully remediated, i.e. no more reading and spelling deficits, (4) non-dyslexic typical readers. Before training, less iFC was observed for dyslexics between two seeds in the left posterior reading network (intraparietal sulcus [L.IPS] and left fusiform gyrus [L.FFG]). After remediation, all dyslexic groups showed weaker iFC between L.IPS and the left middle frontal gyrus. Both remediation groups showed stronger iFC between the L.FFG and right middle occipital gyrus compared to typical readers and non-remediated dyslexics after training. Furthermore, the full remediation group showed stronger negative iFC between L.FFG and the right medial prefrontal cortex. This pattern of brain connectivity at rest suggests that successful remediation of dyslexic children is associated with the emergence of compensatory changes in L.FFG iFC. These studies demonstrate the usefulness of rsfMRI in the analysis of training-related changes in iFC in the brain networks involved in reading.

While the previous studies in dyslexia focused on reading skills, it must be emphasized that dyslexic children and adults also suffer from writing problems [28, 36, 37]. This is true to a greater extent in FI. To date, only one study used resting state with functional illiterates: Skeide et al. [71] observed literacy-induced neural 
plasticity in adult Indian illiterates. Six months of reading instruction lead to increases in the functional connectivity between the occipital lobe and subcortical areas (in the midbrain and thalamus) that were related to individual gains in reading ability.

The present study aimed at exploring changes in iFC at rest associated with reading training in a group of FI adults, thus adding to the previous study of Skeide et al. [71] drawing on subjects raised in an industrialized country. Furthermore, pre-training differences in iFC at rest between the group of FI readers and normal readers were investigated. Twenty adult FI were scanned before and after a 7 month long training of reading and writing abilities (AlphaPlus, [10]). On average, pre-training level of reading was comparable with that of a first-grade student. After training, reading was comparable to a third grade student. Twenty adult normal readers served as a control group.

We used a data-driven ICA-approach to identify resting-state networks. In a second step, we looked for differences in functional connectivity between the two groups before training. For the group of FI, we also looked at the effect of training on functional connectivity. To the extent to which findings in dyslexic readers $[42,43,66,86]$ also apply to FI, we hypothesized decreased functional connectivity in FI in comparison to a group of typical readers in reading-related and nonreading-related neural networks. We also hypothesized that literacy training would normalize functional connectivity in the very same networks that show differences before training (c.f., [48], for a similar study in dyslexia). Specifically, following Skeide et al. [71] literacy training was hypothesized to increase FC between the occipital lobe and subcortical areas.

\section{Methods \\ Participants}

Twenty adult FI participated in the study ( 15 men, mean age 42.70 years, $S E=2.09$ years; range 25 to 58 years, 16 right-handed). They were recruited from an adult literacy program (AlphaPlus, [11, 65]) and received daily training over a period of 7 months using a formal literacy instruction approach combined with computerbased exercises. Although the participants had attended school for several years ( $M=8.95 \pm 0.37$ years), they demonstrated poor literacy skills (see below) and were considered to be functionally illiterate.

The control group comprised 20 adults (15 men, mean age 44.93 years, $S E=3.57$ years; range 27 to 56 years, 16 right-handed). Individuals were included if their reading and writing skills were within a range that can be expected for their age and formal educational status. It was also made sure that they had no former diagnosis of any reading, writing or general learning impairment in childhood.

General inclusion criteria for both groups were: (1) German as the primary language, (2) age above 18 years, (3) general cognitive ability in the normal range and (4) normal hearing and vision. Functional illiterates and control subjects were individually matched for age ( $T$ (39) $=0.72, p=0.47)$ and gender $\left(X^{2}(1)=0, p=1\right)$. The study was conducted in accordance with the declaration of Helsinki. Furthermore, the study protocol was approved by the ethical committee of the University of Bamberg. All participants gave their written informed consent and were paid for test participation $(10 €$ per hour).

\section{Assessment of reading and writing skills}

Reading abilities of functionally illiterate adults were assessed with a standardized German reading test (Würzburger Leise-Leseprobe, WLLP [50]). In this test, 140 written words as well as four pictures next to each word are presented. The participants have to mark the one picture that represents the word on the left side. The test score comprises the number of correctly identified pictures in $5 \mathrm{~min}$. The WLLP is supposed to measure silent reading speed and the ability to decode written words. Writing abilities of functionally illiterate adults were tested with a standardized German writing test for first graders (Diagnostischer Rechtschreibtest, DRT-1 [58]). Here, participants have to write 32 single words from dictation. We used parallel versions of the WLLP and DRT-1 at the beginning as well as at the end of the training in order to assess the efficacy of the training program.

The functional illiterates were scanned prior to and after they participated in a 7 month long reading instruction program (AlphaPlus; see above and [11, 65]).

\section{Literacy training program}

The literacy training program AlphaPlus consists of seven modules that address different aspects of reading and spelling skills. In the present study, course duration was 7 months Monday to Friday. Literacy training duration was approximately 2 to $3 \mathrm{~h}$ daily. Module 1 (classical literacy lessons) consists of four modules with increasing complexity which can be used flexibly in the classroom. For each module, there is a textbook containing specific tasks. These are available online at https:// www.bnw.de/bnwde/content/deutsch/unternehmen/qualifizierung/grundbildung. The audio trainer consists of eight tasks that are designed to train basic perceptual functions in the visual, auditory and motor domain. These perceptual abilities are trained at the beginning of the program; later, only refresher sessions take place. With the help of the Alpha-Trainer (module 3), language 
and perceptual functions are promoted through "lateralized synchronous speech". The trainee vocalizes individual syllables, words, sentences or texts in sync with a model voice. The two different language information (model voice and own voice) are constantly moving from one ear to another in opposite directions (via headphones). In this way, the two voices can be perceived separately and can be compared with each other. In addition, the training elements are presented acoustically as well as visually fostering audio-visual integration as well as the coordination of both brain hemispheres. The training is based on findings suggesting that the corpus callosum is smaller and functionally impaired in individuals with literacy problems [26]. The corpus callosum has been implicated, among other things, in sensory integration processes and in the transfer of information between the hemispheres [62]. The fourth module, the sound discrimination trainer, fosters the distinction between consonants and vowels. The trainee hears short words presented alternately to the left and right ear. Each stimulus begins and ends with a vowel; the middle letter is an alternating consonant (e.g. "eki", "efi") or a letter combination (e.g. "ch", "sch"). By pressing a button, the trainee specifies the perceived middle sound. Module 5 comprises computer-based spelling training using Orthofix ${ }^{\circ}$. On a computer screen, a word is presented, which is either spelled out or read by a model voice. The word disappears after a few seconds and the trainee is prompted to rewrite the word with the keyboard. In simple training mode, word typing is only practiced forward. In the advanced mode, the displayed words have to be typed forward and backward. In its basic form, Orthofix ${ }^{\odot}$ contains about 10.000 of the most common German words, sorted by topic. In addition, there are job-specific word lists that allow the parallel learning of job-specific terminology. Currently, jobrelevant words are implemented for 15 different occupational groups (e. g., professional care, roofers, butchers, landscaping, scaffolders). It is also possible to create own word lists. Another component of the training (module 6) is the online learning platform www.ich-will-lernen.de of the German Adult Education Association. This is Germany's largest literacy learning platform with more than 31.000 different exercises. Course participants worked through individually chosen exercises dependent on their reading and writing level. Module 7 consists of various social training exercises like fitness training, joint cooking, communication training and participation in cultural events.

\section{Image acquisition}

Magnetic-resonance images were acquired on a 3-T Siemens Scanner (Erlangen, Germany) equipped with a standard head coil. A total of $178 \mathrm{~T} 22^{*}$-weighted volumes of the whole brain (EPI-sequence; TR $2000 \mathrm{~ms}$, TE $30 \mathrm{~ms}$, flip angle $80^{\circ}$, FOV $192 \mathrm{~mm}$, matrix $64 \times 64,34$ slices, slice thickness $3 \mathrm{~mm}$, interslice gap $0.75 \mathrm{~mm}$ ) parallel to the anterior-posterior commissural line were recorded for functional imaging resulting in a 6 min run, a duration that has been commonly used in rsfMRI [27].

A T1-weighted high resolution data set was acquired using a 3D-MPRAGE (3 dimensional magnetization prepared rapid acquisition gradient echo) sequence for anatomical information (matrix $192 \times 256,1 \mathrm{~mm}$ isovoxel). The subject's head was fixed during the entire measurement to avoid head movements.

\section{FMRI data analysis}

FMRI analysis at rest was carried out using Multivariate Exploratory Linear Optimized Decomposition into Independent Components (MELODIC) Version 3.14 part of FSL (FMRIB's Software Library, www.fmrib.ox.ac.uk/fsl) [4].

Preprocessing consisted of motion correction, brain extraction, spatial smoothing using a Gaussian kernel of full-width at half maximum (FWHM) of $6 \mathrm{~mm}$, and high-pass temporal filtering equivalent to a timeconstant of $150 \mathrm{~s}(0.007 \mathrm{~Hz})$. For motion detection and correction, six realignment parameters, i.e. three displacements and three elementary rotations, were obtained with respect to the first image in the EPI series. The displacements with respect to the first image of the series were required to be smaller than $3.0 \mathrm{~mm}$ (minimum to maximum) and the individual rotations were required to be smaller than $3.0^{\circ}$. None of the participants had to be excluded according to these criteria. FMRI volumes were registered to the individual's structural scan and standard space images using FMRIB's Nonlinear Image Registration Tool (FNIRT). Preprocessed functional data containing 178 time points for each subject were temporally concatenated across subjects to create a single $4 \mathrm{D}$ data set. The between-subject analysis of the resting data was carried out using a regression technique (dual regression) that allows for voxel-wise comparisons of resting functional connectivity [25]. This approach proceeds in 3 stages. First, the concatenated multiple FMRI data sets are decomposed using ICA to identify large-scale patterns of functional connectivity in the population of subjects. In this analysis, the data set was decomposed into 30 components, in which the model order was estimated using the Laplace approximation to the Bayesian evidence for a probabilistic principal component model [5].

Second, the dual-regression approach is used to identify, within each subject's FMRI data set, subject-specific temporal dynamics and associated spatial maps. This involves first using the full set of group-ICA spatial maps 
in a linear model fit (spatial regression) against the separate fMRI data sets, resulting in matrices describing temporal dynamics for each component and subject, then using these time-course matrices in a linear model fit (temporal regression) against the associated fMRI data set to estimate subject-specific spatial maps.

These spatial maps characterize the subject- and voxel-specific degree of integration into a given group component map. Finally, the different component maps are collected across subjects into single 4D files (1 per original ICA map, with the fourth dimension being subject identification) and tested voxel-wise for statistically significant differences between groups using nonparametric permutation testing [59]. This results in spatial maps characterizing the between-subject/group differences. These maps were thresholded at $p<0.05$ (Familywise error corrected) using Threshold-free cluster enhancement (TFCE [73]) to define clusters of significant changes in connectivity. Data were visualized in MNI standard space using FSLView.

\section{Seed based correlation analysis (SBCA)}

Subject/session-specific measures of voxel-wise subcortical connectivity with each of the RSNs were obtained using Seed Based Correlation Analysis; SBCA tool part of FSL $[15,60]$, for controls and functional illiterates before and after treatment separately. SBCA was carried out within a subject-specific, anatomically-derived subcortical seed mask. Every voxel within each 'individualized' seed mask was quantitatively tested in terms of its connectivity with each of the RSN target maps. We employed two variations on the analysis approach reported previously in a study of healthy volunteers [15], both of which were incorporated to account for the potential (but relatively unclear) contribution of tissue macro- or micro-structural variations (e.g., atrophy, cortical thinning) to regional neuroanatomical changes. Firstly, to ensure more accurate alignment of neural systems defined at a group level to the brain space of individuals, prior to affine transformation to EPI space, RSN target maps from group-ICA were nonlinearly (as opposed to linearly) transformed from MNI space to the high-resolution space of each subject (as implemented in FSL FNIRT); wherein, secondly and prior to registration to EPI space, voxels failing to exceed a comparatively liberal threshold of $>10 \%$ probability of containing grey matter (relative to $>20 \%$ applied previously, as calculated using FSL FAST) in the equivalent T1 structural were removed from these 'subject-specific' RSN spatial maps.

To construct subject-specific subcortical seed masks, T1 structural images were segmented using FSL FIRST. Bilateral regions included in these masks were the entire striatum (comprising regions of caudate, putamen and ventral striatum), pallidum, amygdala, hippocampus and thalamus (and midbrain, see below). The unthresholded versions of these segmented structures (i.e., without boundary correction; see [61] were combined into a single mask image for each subject. To include midbrain voxels within our masks, we carried out nonlinear warp transformation (using FNIRT) of six binary, bilateral volumes (midbrain, substantia nigra, subthalamic nucleus, red nucleus, mammillary body and medial geniculum body) from the Talairach Daemon atlas [52] to the highresolution space of each subject. This midbrain information was then added to the mask containing subjects' other subcortical regions. These subject-specific combined masks were then affine registered to EPI space using FSL FLIRT and used in subsequent subject-wise SBCA, to quantify subcortical functional connectivity with large-scale RSNs.

\section{First-level analysis}

In SBCA the subcortical seed masks from each subject were examined individually, in EPI space, for their voxel-wise spatial distributions of functional connectivity strength with the characteristic activity of each of the RSNs. Voxel-wise connectivity strengths were quantified by calculating partial correlation coefficients between the BOLD signal time series at each mask voxel and that of the weighted principal eigenvariate associated with each RSN (the latter calculated via subject-wise principal component analyses [60]. Voxel-wise coefficients are termed 'partial' because the analysis associated with a given target RSN controlled, in turn, for the seed voxel's activity relationship with each of the other RSNs examined as targets in separate correlation analyses. In these analyses we also controlled for the confounding influences of structured noise from white matter (WM) and cerebrospinal fluid (CSF) tissue types and residual motion artifacts. To this end, binary T1-segmented maps of WM and CSF (calculated using FAST) were registered to EPI space using FLIRT and, for each session, used as masks against the associated, preprocessed functional datasets, in order to extract confound time series that were calculated as the mean BOLD signal within these tissue masks. As well as these WM and CSF confounds, six time series resulting from the motion correction procedure (as implemented in FSL MCFLIRT) describing individual subject head motion parameters were also regressed out of the SBCA. Paired and unpaired t-tests revealed that mean and relative head motion did not differ significantly between training sessions or subject groups (all $p>0.5$ ).

\section{Higher-level analyses}

We used all RSNs in higher-level analyses. The partial connectivity between subcortical seed masks and each 
target was tested voxel-wise for statistically significant differences between groups using nonparametric permutation testing [59]. This results in spatial maps characterizing the between-subject/group differences. These maps were thresholded at $p<0.05$ (FWE corrected) using TFCE [73] to define clusters of significant changes in connectivity. Statistical contrasts in the higher-level analysis specifically examined RSN functional connectivity patterns for differences between controls and functional illiterates and basic paired effects of treatment.

To investigate interactions between trainingdependent neurobiological effects and psychological scores revealed in this study, bivariate correlations (Pearson's $\mathrm{r}, p<0.05$, two-tailed) were carried out between individual subject measures of functional connectivity in different networks and psychological parameters. Significant clusters identified from the difference between preand post-training were thus used as masks to extract mean values from individual data sets. The differences in these values resulting from training and that of psychometric parameters were used to test for correlation.

\section{Results}

\section{Reading and writing skills}

Literacy scores for both groups are presented in Table 1. With regard to reading, the FI group showed significant improvement due to the training ( $\mathrm{t}(19)=3.78, p<0.01$ ), but still did not reach the reading level of the control participants after training ( $\mathrm{t}(30)=8.00, p<0.001$ ). Likewise, the number of writing errors significantly decreased in the FI group due to the training $(\mathrm{t}(19)=6.25$, $p<0.001)$. Again, their error level after training was considerably higher than that of the control group $(\mathrm{t}(30)=$ $4.14, p<0.001)$.

\section{Independent component analysis}

The ICA decomposition resulted in 30 spatial maps, containing classically identified resting-state networks [4, $19,21]$ as well as artefactual components. The complete set of 30 components was used for the dual-regression analysis. Only three of the original components showed between-group changes in the degree of functional connectivity: the left Fronto-Parietal Network (LFPN), the Visual Network (VN) and the Basal Ganglia Network (BGN).

\section{Left Fronto-parietal network (LFPN)}

Two lateralized frontoparietal networks (left: LFPN, right: RFPN) were identified, each comprising anterior insula, medial frontal cortex, and lateral frontal and parietal regions. Only the LFPN showed increased connectivity in FI in middle frontal gyrus and frontal orbital cortex compared to the control group (Fig. 1; Table 2).

\section{Basal ganglia network (BGN)}

This network included thalamus, caudate, putamen, pallidum, amygdala, insular cortex, central opercular cortex, supplementary motor cortex and cingulate gyrus. The group analysis showed increased functional connectivity between right supramarginal gyrus, posterior division and this network in FI (Fig. 2a; Table 2).

\section{Visual networks (VN)}

ICA identified two visual cortical sub-networks: the first consisting of temporal occipital fusiform gyrus, lateral occipital cortex, occipital pole, lingual gyrus and thalamus (VN1); and the second comprising intracalcarine cortex, lingual gyrus, occipital pole, occipital fusiform gyrus and cuneal cortex (VN2). We found a significantly decreased functional connectivity of left lateral occipital cortex in VN1 in FI and also decreased connectivity of left insular cortex to this network in same group (Fig. $2 \mathrm{~b}$; Table 2). The between group analysis showed no significant changes in VN2.

\section{SBCA}

At this step we focused our analysis on functional connectivity between different subcortical areas (including the entire striatum comprising the caudate nucleus, $\mathrm{pu}-$ tamen and ventral striatum, pallidum, amygdala, hippocampus, thalamus and midbrain) and neocortical networks found in this study. The between group analysis showed changes of connectivity between distinct subcortical areas and neocortical networks including default mode network (DMN), dorsal attention network (DAN), ventral attention network (VAN) and LFPN.

The DMN comprised prefrontal, anterior and posterior cingulate, lateral parietal, and inferior/middle temporal gyri (extending to the mesial temporal lobe), cerebellar areas, and thalamic nuclei. The ICA divided this network in three sub networks including anterior DMN, ventral DMN and posterior DMN. The between

Table 1 Literacy skills of functional illiterates and controls (mean and SD)

\begin{tabular}{llll}
\hline & Functional illiterates $(n=20)$ & & Controls $(n=20)$ \\
\hline Reading skill (correct words) & before training & $39.30(5.85)$ & $135.25(7.66)$ \\
& after training & $55.20(7.66)$ & $16.10(2.36)$ \\
Writing skill (errors) & before training & $10.25(1.91)$ & $0.00(0.00)$ \\
& after training & 10. & \\
\hline
\end{tabular}




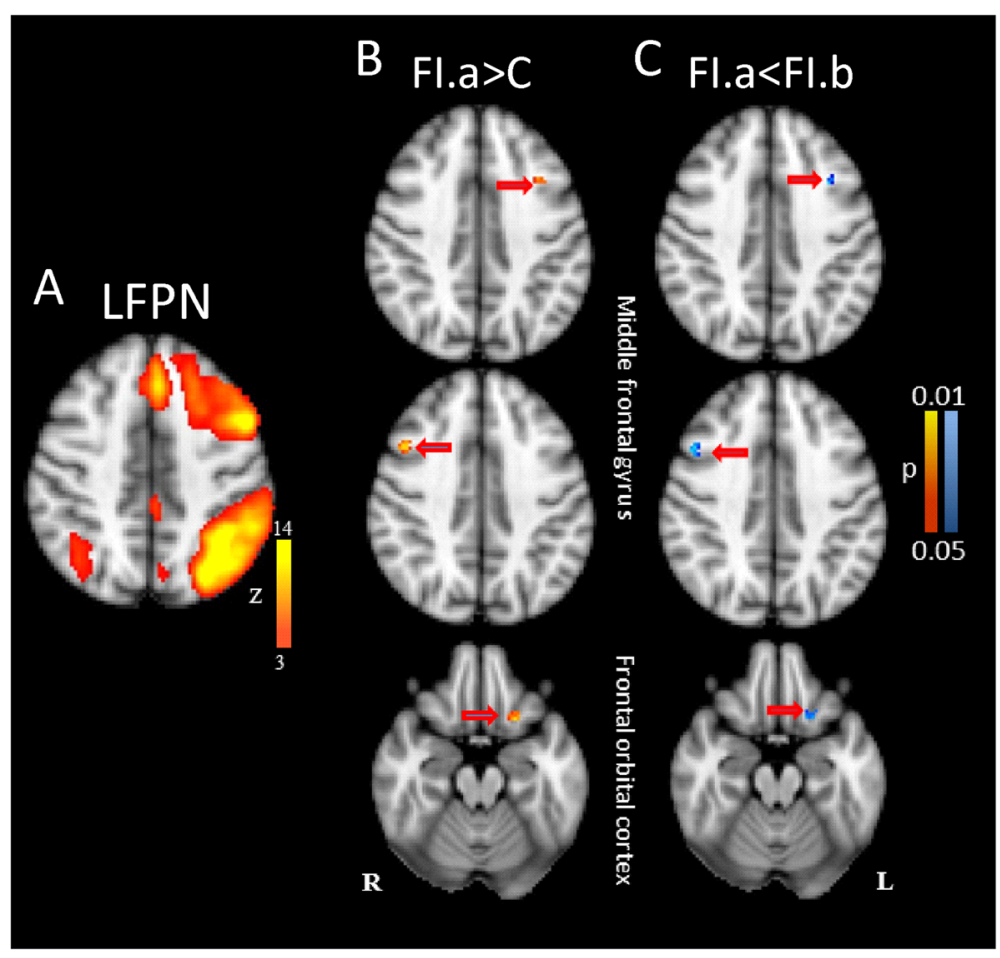

Fig. 1 a left Fronto-Parietal Network (LFPN) as revealed by ICA. b Between group comparison in LFPN comparing functional illiterates before training (Fl.a) and control group (C). b Training induced changes in LFPN in the Fl group: Training led to a normalization. All data are FWEcorrected for multiple comparisons $(p<0.05)$

group analysis showed reduced connectivity between anterior DMN and left amygdala/right hippocampus in FI (Fig. 3a, Table 3). However, the connectivity was increased between ventral DMN and a cluster spanning right putamen and right $\mathrm{Ncl}$. accumbens in the same group (Fig. 3g, Table 3).

The VAN consisted of precuneus cortex, cingulate gyrus, angular gyrus, superior frontal gyrus and middle frontal gyrus and showed reduced connectivity to the right hippocampus (Fig. 3e, Table 3) in FI, relative to controls. The DAN was divided into two subnetworks; DAN-I comprising lateral occipital cortex, superior parietal lobule, supramarginal gyrus, inferior temporal gyrus, superior frontal gyrus, inferior frontal gyrus, precentral gyrus and postcentral gyrus and DAN-II with extension to dorsal precuneus. We found significantly

Table 2 Regions with changed functional connectivity in functional illiterates (FI) before training $(n=20)$ compared to controls in different networks

\begin{tabular}{|c|c|c|c|c|c|c|}
\hline \multirow[t]{2}{*}{ Brain region } & \multirow[t]{2}{*}{ Hemisphere } & \multicolumn{3}{|c|}{ MNI coordinates } & \multirow{2}{*}{$\begin{array}{l}\text { Cluster } \\
\text { size }\end{array}$} & \multirow{2}{*}{$\begin{array}{l}p \\
\text { (FWE) }\end{array}$} \\
\hline & & $x$ & Y & Z & & \\
\hline \multicolumn{7}{|l|}{ Controls $<$ FI before training } \\
\hline \multicolumn{7}{|l|}{ LFPN } \\
\hline Middle Frontal Gyrus & L & -38 & 14 & 40 & 24 & 0.03 \\
\hline Middle Frontal Gyrus & $\mathrm{R}$ & 46 & 16 & 40 & 55 & 0.02 \\
\hline Frontal Orbital Cortex & L & -22 & 20 & -22 & 30 & 0.02 \\
\hline \multicolumn{7}{|l|}{ BG Network } \\
\hline Supramarginal Gyrus, posterior division & $\mathrm{R}$ & 56 & -40 & 26 & 24 & 0.03 \\
\hline \multicolumn{7}{|l|}{ Controls $>$ Fl before training } \\
\hline \multicolumn{7}{|l|}{ Visual Network } \\
\hline Lateral Occipital Cortex, inferior division & L & -36 & -78 & -4 & 26 & 0.03 \\
\hline Insular Cortex & L & -34 & 14 & -12 & 84 & 0.02 \\
\hline
\end{tabular}




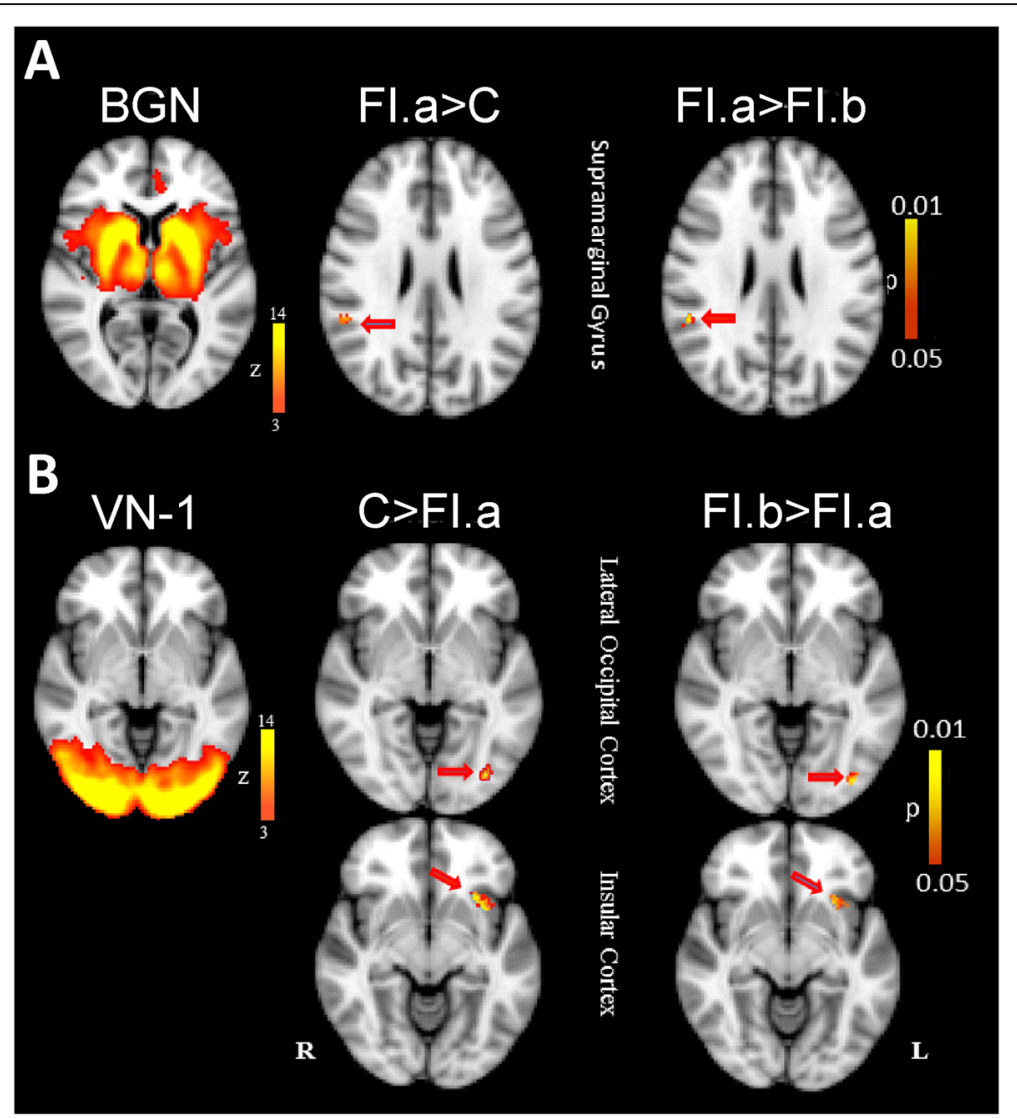

Fig. 2 a basal ganglia network (BGN, left); between group comparison comparing Fl before training (Fl.a) and control group (C) (middle); training effect comparing Fl before (Fl.a) and after (Fl.b) training (right). b Visual Network-I (VN-1, left column); between group comparison comparing FI before training (Fl.a) and control group (C) (middle); training effect comparing Fl before (Fl.a) and after (Fl.b) training (right). All data are FWEcorrected for multiple comparisons $(p<0.05)$

reduced connectivity between DAN-II and the left putamen (Fig. 3b, Table 3) in FI, relative to controls.

Furthermore, we found reduced connectivity between VN1 and right thalamus (Fig. 3d, Table 3) and between LFPN right putamen (Fig. 3c, Table 3) in FI but increased connectivity of LFPN to right thalamus (Fig. 3f, Table 3).

\section{Training effects}

The gain in reading and writing abilities in FI was associated with the following neural changes: In LFPN the training resulted in the reversal of 'hyperconnectivity' (relative to control subjects) in FI in middle frontal gyrus and frontal orbital cortex (Fig. 1, Table 4). The hyperconnectivity between supramarginal gyrus and BGN in FI was reversed after training (Fig. 2a, Table 4). Considering VN1 we found increased functional connectivity in lateral occipital cortex and insular cortex after training, reaching the same level as in healthy controls (Fig. 2b, Table 4).

The connectivity between subcortical areas and different networks in FI shown above was also influenced by training (Fig. 3, Table 5): training increased the connectivity between anterior DMN and left amygdala/right hippocampus and between VAN and left thalamus. However, we found a significant decrease of connectivity between LFPN and right thalamus and between ventral $\mathrm{DMN}$ and right putamen/right Ncl. accumbens.

We also performed a comparison between the imaging data of controls and illiterates after literacy training. These analyses did not reveal significant effects at the chosen statistical threshold. Decreasing the statistical threshold revealed a pattern similar to the comparison between controls and illiterates before training.

\section{Correlation to reading and writing performance}

The reversal of hyperconnectivity (i.e. the connectivity difference between pre- and posttraining sessions) of right middle frontal gyrus to the LFPN and of right supramarginal gyrus to the BGN correlated positively with reading ability as assessed by WLLP and negatively with the number of writing errors as assessed by DRT-1. The increase of functional connectivity in left insular 


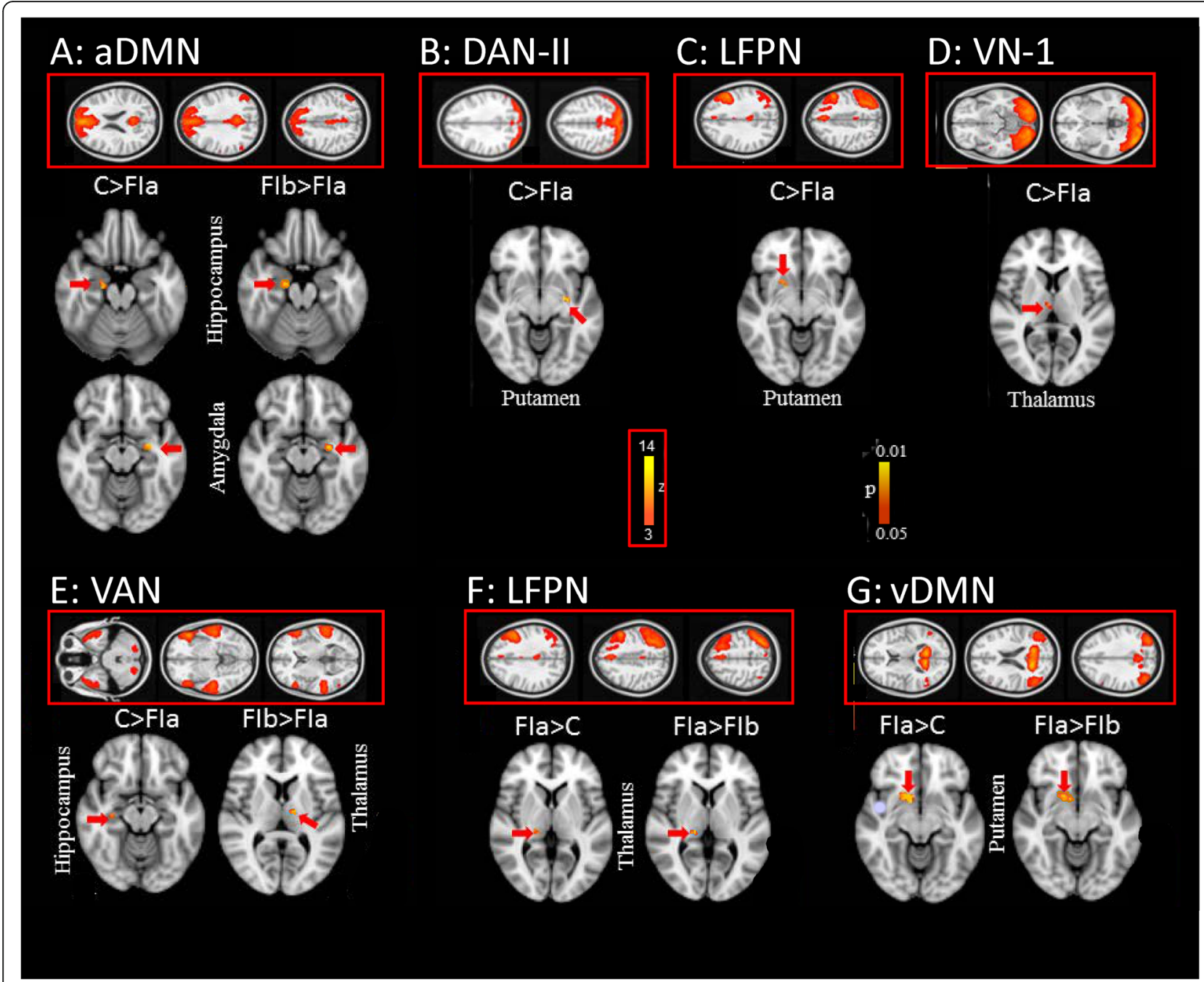

Fig. 3 Seed Based Correlation Analysis (SBCA) between selected subcortical areas (indicated by red arrows) and different neocortical networks. a anterior default mode network (aDMN); b dorsal attention network II (DAN-II); $\mathbf{c} / \mathbf{f}$ left fronto-parietal network (LFPN); $\mathbf{d}$ visual network-I (VN-1); e ventral attention network; $\mathbf{g}$ ventral default mode network (vDMN). All data are FWE-corrected for multiple comparisons $(p<0.05)$. C: Control group; Fl.a: functional illiterates before training; Fl.b: functional illiterates after training

Table 3 Changed connectivity between different subcortical and neocortical networks compared between Controls and functional illiterates (FI) before training

\begin{tabular}{|c|c|c|c|c|c|c|c|}
\hline \multirow[t]{2}{*}{ Brain region } & \multirow[t]{2}{*}{ Hemisphere } & \multirow[t]{2}{*}{ IC/Network } & \multicolumn{3}{|c|}{ MNI coordinates } & \multirow{2}{*}{$\begin{array}{l}\text { Cluster } \\
\text { size }\end{array}$} & \multirow{2}{*}{$\begin{array}{l}p \\
\text { (FWE }\end{array}$} \\
\hline & & & $\bar{x}$ & Y & z & & \\
\hline \multicolumn{8}{|c|}{ Controls > Fl before training } \\
\hline Hippocampus & $\mathrm{R}$ & anterior DMN & 20 & -12 & 20 & 53 & 0.02 \\
\hline Amygdala & L & anterior DMN & -28 & -8 & -16 & 30 & 0.01 \\
\hline Putamen & L & DAN (Precuneus) & -30 & -10 & -6 & 27 & 0.01 \\
\hline Putamen & R & LFPN & 20 & 10 & -8 & 19 & 0.01 \\
\hline Thalamus & $\mathrm{R}$ & Visual-II & 6 & -14 & 6 & 16 & 0.03 \\
\hline Hippocampus & R & VAN & 30 & -20 & -14 & 95 & 0.01 \\
\hline \multicolumn{8}{|c|}{ Fl before training $>$ Controls } \\
\hline Thalamus & R & LFPN & 16 & -28 & 4 & 15 & 0.02 \\
\hline Putamen & R & ventral DMN & 18 & 10 & -10 & 98 & 0.01 \\
\hline Accumbens & R & ventral DMN & 10 & 10 & -8 & 47 & 0.01 \\
\hline
\end{tabular}


Table 4 Regions with changed functional connectivity in functional illiterates after training $(n=20)$ compared to before training in different networks

\begin{tabular}{|c|c|c|c|c|c|c|}
\hline \multirow[t]{2}{*}{ Brain region } & \multirow[t]{2}{*}{ Hemisphere } & \multicolumn{3}{|c|}{ MNI coordinates } & \multirow{2}{*}{$\begin{array}{l}\text { Cluster } \\
\text { size }\end{array}$} & \multirow{2}{*}{$\begin{array}{l}p \\
\text { (FWE }\end{array}$} \\
\hline & & $\bar{x}$ & $\mathrm{Y}$ & Z & & \\
\hline \multicolumn{7}{|l|}{ After < Before training } \\
\hline \multicolumn{7}{|l|}{ LFPN } \\
\hline Middle Frontal Gyrus & L & -36 & 14 & 40 & 10 & 0.03 \\
\hline Middle Frontal Gyrus & $\mathrm{R}$ & 44 & 14 & 42 & 23 & 0.03 \\
\hline Frontal Orbital Cortex & L & -22 & 22 & -22 & 26 & 0.02 \\
\hline \multicolumn{7}{|l|}{ BG Network } \\
\hline Supramarginal Gyrus, posterior division & $\mathrm{R}$ & 56 & -40 & 24 & 17 & 0.03 \\
\hline \multicolumn{7}{|l|}{ After $>$ Before training } \\
\hline \multicolumn{7}{|l|}{ Visual Network } \\
\hline Lateral Occipital Cortex, inferior division & L & -36 & -82 & -4 & 12 & 0.03 \\
\hline Insular Cortex & L & -32 & 16 & -10 & 53 & 0.03 \\
\hline
\end{tabular}

cortex in VN1 also correlated positively with WLLP and negatively with writing errors (Table 6).

With regard to SBCA we found that increased connectivity between left amygdala and anterior DMN in FI after training correlated positively with WLLP (Pearson coefficient $0.68, p<0.001$ two tailed). We found no other correlations considering WLLP or DRT-1.

\section{Discussion}

In this study we used rs-fMRI to elucidate differences in intrinsic functional connectivity between FI and normal readers and their response to intensive literacy training. Training led to a significant improvement of reading and writing skills. In line with our hypotheses, the differences in reading between normal readers and the FI group as well as the training-induced changes in reading performance were accompanied by changes of intrinsic connectivity of the brain at rest, i.e. when the participants were not required to read. In other words, the degree of reading ability leaves its traces in brain connectivity during the idling state of the brain.
To summarize, the between group analysis showed increased iFC in FI in the LFPN (anterior insula, medial frontal cortex, lateral and frontal parietal regions) and in the BGN (thalamus, caudate, putamen, pallidum, amygdala, supplementary motor cortex and cingulate gyrus) before training. Furthermore, VN1 (encompassing temporal occipital fusiform gyrus, lateral occipital cortex, occipital pole, lingual gyrus, thalamus) showed decreased iFC in FI.

Functional connectivity between the anterior component of the DMN and left amygdala / right hippocampus, between the DAN and the left putamen, between VAN and the right hippocampus as well as between $\mathrm{VN} 1$ and the right thalamus was reduced in FI. An increase in iFC in FI was found between ventral DMN and right putamen/right Ncl. accumbens and between LFPN and the right thalamus. Training-related changes of functional connectivity in the FI group comprised of reversal of the "hyperconnectivity" in middle frontal gyrus and in the frontoorbital cortex and between supramarginal gyrus and the BGN. Furthermore, functional connectivity increased in FI in the VN1 (lateral occipital

Table 5 Subcortical areas with significant changes of connectivity to different networks in functional illiterates after training

\begin{tabular}{|c|c|c|c|c|c|c|c|}
\hline \multirow[t]{2}{*}{ Brain region } & \multirow[t]{2}{*}{ Hemisphere } & \multirow[t]{2}{*}{ Network } & \multicolumn{3}{|c|}{ MNI coordinates } & \multirow{2}{*}{$\begin{array}{l}\text { Cluster } \\
\text { size }\end{array}$} & \multirow{2}{*}{$\begin{array}{l}p \\
\text { (FWE }\end{array}$} \\
\hline & & & $\bar{x}$ & $\mathrm{Y}$ & Z & & \\
\hline \multicolumn{8}{|c|}{ After training $>$ before training } \\
\hline Hippocampus & $\mathrm{R}$ & anterior DMN & 22 & -16 & -20 & 67 & 0.02 \\
\hline Amygdala & L & anterior DMN & -26 & -8 & -16 & 18 & 0.01 \\
\hline Thalamus & L & VAN & -10 & -12 & 8 & 74 & 0.01 \\
\hline \multicolumn{8}{|c|}{ Before training $>$ after training } \\
\hline Thalamus & $\mathrm{R}$ & LFPN & 16 & -28 & 2 & 14 & 0.03 \\
\hline Putamen & $\mathrm{R}$ & ventral DMN & 18 & 10 & -10 & 62 & 0.01 \\
\hline Accumbens & $\mathrm{R}$ & ventral DMN & 10 & 10 & -10 & 28 & 0.02 \\
\hline
\end{tabular}


Table 6 Regions with changed functional connectivity in functional illiterates after training $(n=20)$ compared to before training in which we found significant correlation ( $p<0.005$, two tailed) to WLLP and DRT1. The table shows the Pearson coefficient ( $r$ )

\begin{tabular}{lllll}
\hline Brain region & Hemisphere & Network & Reading (WLLP) & Writing (DRT-1) \\
\hline Middle Frontal Gyrus & $\mathrm{R}$ & LFPN & 0.48 & -0.47 \\
Insular Cortex & $\mathrm{L}$ & Visual & 0.49 & -0.45 \\
Supramarginal Gyrus, posterior division & $\mathrm{R}$ & $\mathrm{BG}$ & 0.46 & -0.44 \\
\hline
\end{tabular}

cortex, insular cortex). These changes in connectivity correlated with gains in reading speed and spelling accuracy.

These findings add to the growing evidence that on a neural level, problems of reading and writing are reflected in differences in brain connectivity mainly involving the neural networks that support the reading process (e.g., [56, 83, 85]). Furthermore, learning to read and write in adulthood leads to changes in iFC in several brain regions relevant for reading (see also [71]).

It is important to emphasize again, that the current analysis is based on resting state fMRI recordings. Any differences between literate participants and FI participants may thus be either driven by the inability of the FI participants to read and / or by compensatory mechanisms engaged in the FI participants. For example, the inability to read my cause FI participants to rely on memory processes to a greater extent. Therefore, the fact that we found normalization of the connectivity differences due to training is very important and suggests that the differences between FI and typical reading participants are mainly due to their inability to read.

\section{Pre-training group differences in iFC in resting-state neural networks}

The FPN is involved in rule-based problem solving and goal-directed behavior using and manipulating working memory $[46,55,57,63]$. This network is thought to support online task-control that allows rapid adaptation of control settings from one event to the next. It is referred to as "task positive" because it increases in activity when attention is directed to external stimuli in cognitive tasks $[75,81]$. Two lateralized FPNs with predominantly left (LFPN) and predominantly right (RFPN) brain areas involved can be distinguished. Both networks also comprise minor contributions from brain areas in the contralateral hemisphere (c.f [49].), as was also case in the current data set. According to a meta-analysis of the BrainMap database, RFPN activity associates predominantly with action inhibition, cognition and memory. However, in addition to cognitive memory functions the LFPN has also been found to associate significantly with processes subserving language-related cognition [72]. For example, this network is activated during tasks involving words, pseudowords, letters, or Asian characters [51]. The increased functional connectivity of LFPN in the FI group may reflect compensatory hyperconnectivity of this network involved in online task control and different aspects of language in untrained subjects. Alternatively, this hyperconnectivity may also reflect disease-related inhibition between networks. In any case, hyperconnectivity was normalized after the training, as indicated by the fact that the comparison between controls and illiterates after training did not reveal significant effects for the network. In line with these findings Horowitz-Kraus et al. [42, 43] reported training-dependent changes in attentional and cognitive control networks encompassing the middle frontal gyrus in a sample of dyslexic children aged 8-12 years.

The Visual Networks include areas that are known to be central to different aspects of neural processing within the visual hierarchy. The Visual Network-I in this study corresponds spatially to a network identified in BrainMap meta-analyses that additionally associates with language and orthography, which may reflect the perception of forms in written language and reading [51, 72]. In untrained FI we found Visual Network-I to display decreased connectivity with lateral occipital cortex and also with insular cortex. Both of these regions are also involved in language processing and may show decreased connectivity when under-utilized. Insula is supposed to be strongly involved in perception and awareness. Different task-related studies show increased activity in the insula as a component of various largescale networks across a variety of cognitive tasks (for review see [76];). Decreased functional connectivity between insula and visual network in this study may mirror reduced awareness/perception of written language as meaningful visual stimuli in untrained FI [12]. This was reversed by training and the post-training increase of functional connectivity between left insular cortex and this network correlated positively with WLLP and negatively with DRT-1, which suggests increased awareness/ perception of written language.

Besides its involvement in motor tasks several studies have shown that the basal ganglia network is involved in various reading and language tasks $[13,51,78]$. The results of BrainMap meta-analyses reveal functional networks activated during different tasks from more than 16,000 fMRI scans. These task-dependent networks strongly correspond to resting state networks, such as those found in this study. It is thus supposed that the 
same areas activated in a network during a task remain highly functionally connected with each other at rest [72].

\section{Group differences in functional connectivity between different subcortical areas}

At first glance, the clear implication of subcortical regions in the current analysis may seem somewhat counterintuitive. However, as already pointed out in the introduction, subcortical regions including the putamen, striatum and thalamus have been shown to be related to reading previously (). For example, Liu et al. [54] compared reading networks in children and adults and found a greater involvement of subcortical structures in children. According to these author, this "may indicate that children rely on sensorimotor circuits and their connection to cortical linguistic brain regions to maintain sound representations for reading" [54]. The putamen has been suggested to be engaged in the cortical initiation of phonological processing during reading [13].

In the present study, the (ICA-characterized) basal ganglia network itself showed no significant betweengroup differences. We found only increased functional connectivity between this network and right supramarginal gyrus (posterior division) in untrained FI, which was reversed after training and correlated with WLLP and DRT-1. The left supramarginal gyrus is part of a language network in right-handed subjects. The right one has been found to be involved in empathy [70] and phonological decisions [35]. However, there is evidence for reorganization of this area as a part of a language network after recovery from stroke or operation [38]. It is supposed that the predominant left side of the language network inhibits the right side and after loss of this inhibition the right network could be activated [40, 44]. Whether the increased functional connectivity between right supramarginal gyrus and basal ganglia in untrained FI reflects compensation resulting from daily encountering of linguistic problems as permanent stimuli remains questionable. However, in line with this interpretation this state was normalized after training correlating with improvements in WLLP and DRT-1 scores.

Finally, we investigated functional connectivity of other subcortical areas in terms of their correlation with different networks as a whole to assess the role of largescale cortico-subcortical interplay in language learning and plasticity. DMN is known to be important for episodic, autobiographical and semantic memory, selfrelated and social cognitive processes, value-based decision making and emotion regulation [14, 20,74]. Recent studies showed activation or deactivation of different parts of DMN during different language tasks, emphasizing its role in language understanding and speech production in healthy subjects [6, 67]. We found both reduced and increased functional connectivity between different subcortical areas and different parts of the DMN.

\section{Effect of literacy training on iFC}

Importantly, training-related gains in literacy skills were accompanied by changes in functional connectivity in the FI group that by and large can be characterized in terms of a partial normalization, i.e. the connectivity patterns were changed after training to be closer to the normal reading control group.

For example, the changes of DMN observed in FI were partly reversed after training, which furthermore correlated with improvement of WLLP scores. This shows the relevance of the interaction between DMN and these subcortical areas for language processing.

In addition, the increased connectivity between LFPN and right thalamus in untrained FI was reversed after training, again suggesting a normalizing effect of literacy training on functional connectivity. The role of thalamus and basal ganglia in language processing is complex and has been discussed intensively by Klostermann et al. [45]. The thalamus is a relay area involved in the switching between different cortical areas/networks involved in speech, depending on functional demands. It receives information from one part of a network and communicates it further to areas at different levels of the processing hierarchy, to rapidly and selectively facilitate goal-oriented functions, for example word generation. The differences in connectivity between putamen and thalamus and LFPN may reflect group differences in the interaction/influence of thalamus and basal ganglia with respect to cortical areas needed for language production. Finally, the reduced connectivity between Visual Network-I and right thalamus may show reduced visual and attentional interplay by untrained FI due to language.

\section{Limitations}

As any method, rs-fMRI comes with intrinsic limitations. The situation during rest may be considered as insufficiently controlled [77]. Also resting state activity fails to uncover changes in task strategies or functional compensation that occur only during performance. Analysis of resting state activity therefore should be considered as complementary to functional measures. The next important point is the problem of motion in all fMRI studies. In our study we found no increased motion in both groups and no significant differences between both groups. However, using ICA can further minimize effect of motion on results as described elsewhere in details [16]. Especially it could be shown that ICA methods can reliably identify and account for the artefactual influence 
of respiratory and cardiovascular signal fluctuation on found networks $[4,7]$.

\section{Conclusion}

In summary the data presented here show significant changes of functional connectivity in cortical and subcortical areas which partly change after training and correlate with increases in reading skills. Whether any or a number of these areas are candidate for non-invasive brain stimulation like repetitive transcranial magnetic stimulation (rTMS) of transcranial direct current stimulation (tDCS) needs further studies [17, 18, 39, 84]. Here, the question is, if manipulation of the resting state of the brain following stimulation of distinct nodes of networks (e.g., Visual Network-I, DMN or VAN) helps better learning.

\section{Abbreviations}

BGN: Basal Ganglia network; CSF: Cerebrospinal fluid; DAN: Dorsal attention network; DMN: Default mode network; DRT: Diagnostischer Rechtschreibtest; EPI: Echo-planar imaging; FFG: Fusiform gyrus; Fl: Functional illiterate; FWHM: Full-width at half maximum; ICA: Independent component analysis; iFC: intrinsic functional connectivity; IPS: Intraparietal sulcus; LFPN: Leftfronto-parietal network; LIFG: Left inferior frontal gyrus; LMTG: Left middle temporal gyrus; MELODIC: Multivariate Exploratory Linear Optimized Decomposition into Independent Components; MPRAGE: Magnetization Prepared Rapid Acquisition Gradient Echo; rsfMRl: resting state functional magnetic resonance imaging; RSN: Resting state network; SBCA: Seed Based Correlation Analysis; STG: Superior temporal gyrus; TFCE: Threshold-free cluster enhancement; VAN: Ventral attention network; VN1: Visual Network-1; WLLP: Würzburger Leise-Leseprobe; WM: White matter

\section{Acknowledgements}

We thank Annegret Aulbert-Siepelmeyer, Klaus Menkhaus and Ralph Warnke for help during various stages of this project.

\section{Authors' contributions}

$B M, J R$ and $M B$ designed the study. $M B$ and $B M$ acquired the data. BM and $D C$ analyzed the data. BM, MB, JR, TFM wrote the paper. The author(s) read and approved the final manuscript.

\section{Funding}

Deutsche Forschungsgemeinschaft (SFB TR134 project C1) awarded to TFM Bundesministerium für Bildung und Forschung (01AB12032C) awarded to JR.

\section{Availability of data and materials}

Data will be freely shared by the authors.

\section{Ethics approval and consent to participate}

The study was approved the Ethics committee of the University of Bamberg. All participants gave informed consent prior to participation.

\section{Consent for publication}

Not applicable.

\section{Competing interests}

The authors declare that they have no competing interests in this research.

\section{Author details}

${ }^{1}$ CNS-LAB, International Neuroscience Institute (INI), Hannover, Germany. ${ }^{2}$ Department of Neurology, University of Lübeck, Ratzeburger Allee 160 23562 Lübeck, Germany. ${ }^{3}$ Institute of Psychology II, University of Lübeck, Lübeck, Germany. ${ }^{4}$ Translational Neuromodeling Unit, Institute for Biomedical Engineering, University of Zurich \& ETH Zurich, Zurich, Switzerland. ${ }^{5}$ Department of Psychology, University of Bamberg, Bamberg, Germany. ${ }^{6}$ Bamberg Graduate School of Cognitive and Affective Sciences (BAGrACS),
Bamberg, Germany. ${ }^{7}$ Neurologische Klinik Hessisch Oldendorf, Hessisch Oldendorf, Germany.

Received: 27 March 2019 Accepted: 22 April 2020

Published online: 12 May 2020

\section{References}

1. Alcauter, S., García-Mondragón, L., Gracia-Tabuenca, Z., Moreno, M. B., Ortiz, J. J., \& Barrios, F. A. (2017). Resting state functional connectivity of the anterior striatum and prefrontal cortex predicts reading performance in school-age children. Brain and Language, 174, 94-102.

2. ANLCI. (2018). L'évolution de l'illettrisme en France. Lyon: Agence Nationale de Lutte Contre l'lllettrisme.

3. Banai, K., Hornickel, J., Skoe, E., Nicol, T., Zecker, S., \& Kraus, N. (2009). Reading and subcortical auditory function. Cerebral Cortex, 19, 2699-2707.

4. Beckmann, C. F., DeLuca, M., Devlin, J. T., \& Smith, S. M. (2005). Investigations into resting-state connectivity using . component analysis. Philosophical Transactions of the Royal Society of London. Series B, Biological Sciences, 360, 1001-1013.

5. Beckmann, C. F., \& Smith, S. M. (2004). Probabilistic independent component analysis for functional magnetic resonance imaging. IEEE Transactions on Medical Imaging, 23, 137-152.

6. Binder, J. R., Desai, R. H., Graves, W. W., \& Conant, L. L. (2009). Where is the semantic system? A critical review and meta-analysis of 120 functional neuroimaging studies. Cerebral Cortex, 19, 2767-2796.

7. Birn, R. M., Murphy, K. \& Bandettini, P. A. (2008). The effect of respiration variations on independent component analysis results of resting state functional connectivity. Human Brain Mapping, 29, 740-750.

8. Biswal, B., Yetkin, F. Z., Haughton, V. M., \& Hyde, J. S. (1995). Functional connectivity in the motor cortex of resting human brain using echo-planar MRI. Magnetic Resonance in Medicine, 34, 537-541.

9. Bolger, D. J., Perfetti, C. A., \& Schneider, W. (2005). Cross-cultural effect on the brain revisited: Universal structures plus writing system variation. Human Brain Mapping, 25, 92-104

10. Boltzmann, M. Rüsseler, J., Ye, Z, \& Münte, T. F. (2013). Learning to read in adulthood: An evaluation of a literacy program for functionally illiterate adults in Germany. Problems of Education in the 21st Century, 51, 33-46.

11. Boltzmann, M., Aulbert-Siepelmeyer, A., Rüsseler, J., Warnke, R., Menkhaus, K., \& Overlander, O. (2015). AlphaPlus - Ein Alphabetisierungsprogramm zur Förderung der Schriftsprachkompetenz Erwachsener. Bielefeld: W Bertelsmann Verlag.

12. Boltzmann, M., \& Rüsseler, J. (2013). Training-related changes in early visual processing of functionally illiterate adults: Evidence from event-related brain potentials. BMC Neuroscience, 14, 154

13. Booth, J. R., Wood, L., Lu, D., Houk, J. C., \& Bitan, T. (2007). The role of the basal ganglia and cerebellum in language processing. Brain Research, 1133, 136-144.

14. Christoff, K., Gordon, A. M., Smallwood, J., Smith, R., \& Schooler, J. W. (2009). Experience sampling during $\mathrm{fMRI}$ reveals default network and executive system contributions to mind wandering. Proceedings of the National Academy of Sciences of the United States of America, 106, 8719-8724.

15. Cole, D. M., Oei, N. Y., Soeter, R. P., Both, S., van Gerven, J. M., Rombouts, S. A., \& Beckmann, C. F. (2013). Dopamine-dependent architecture of corticosubcortical network connectivity. Cerebral Cortex, 23, 1509-1516.

16. Cole, D. M., Smith, S. M., \& Beckmann, C. F. (2010). Advances and pitfalls in the analysis and interpretation of resting-state FMRI data. Frontiers in Systems Neuroscience, 4, 8.

17. Costanzo, F., Menghini, D., Caltagirone, C., Oliveri, M., \& Vicari, S. (2013). How to improve reading skills in dyslexics: The effect of high frequency rTMS. Neuropsychologia, 51, 2953-2959

18. Costanzo, F., Varuzza, C., Rossi, S., Sdoia, S., Varvara, P., Oliveri, M., Giacomo, K. Vicari, S., \& Menghini, D. (2016). Evidence for reading improvement following tDCS treatment in children and adolescents with dyslexia. Restorative Neurology and Neuroscience, 34, 215-226.

19. Damoiseaux, J. S., Rombouts, S. A., Barkhof, F., Scheltens, P., Stam, C. J. Smith, S. M., \& Beckmann, C. F. (2006). Consistent resting-state networks across healthy subjects. Proceedings of the National Academy of Sciences of the United States of America, 103, 13848-13853.

20. D'Argembeau, A., Collette, F., Van der Linden, M., Laureys, S., Del Fiore, G., Degueldre, C., Luxen, A. \& Salmon, E. (2005). Self-referential reflective activity and its relationship with rest: A PET study. Neuroimage, 25, 616-624. 
21. De Luca, M., Beckmann, C. F., De Stefano, N., Matthews, P. M., \& Smith, S. M. (2006). fMRI resting state networks define distinct modes of long-distance interactions in the human brain. Neuroimage, 29, 1359-1367.

22. Döbert, M., \& Hubertus, P. (2000). Ihr Kreuz ist die Schrift. Analphabetismus und Alphabetisierung in Deutschland. Stuttgart: Bundesverband Alphabetisierung e.V.

23. Egloff, B., Grosche, M., Hubertus, P., Rüsseler, J. (2011). Funktionaler Analphabetismus: Eine aktuelle Definition. In: Projektträger im DLR e.V. (ed.) Zielgruppen in Alphabetisierung und Grundbildung Erwachsener: Bestimmung, Verortung, Ansprache. W. Bertelsmann Verlag, Bielefeld.

24. Eme, E. (2011). Cognitive and psycholinguistic skills of adults who are functionally illiterate: Current state of research and implications for adult education. Applied Cognitive Psychology, 25, 753-762.

25. Filippini, N., MacIntosh, B. J., Hough, M. G., Goodwin, G. M., Frisoni, G. B., Smith, S. M., Matthews, P. M., Beckmann, C. F., \& Mackay, C. E. (2009). Distinct patterns of brain activity in young carriers of the APOE-epsilon4 allele. Proceedings of the National Academy of Sciences of the United States of America, 106, 7209-7214.

26. Fine, J. G., Semrud-Clikeman, M., Keith, T. Z., Stapleton, L. M., \& Hynd, G. W. (2007). Reading and the corpus callosum: An MRI family study of volume and area. Neuropsychology, 21, 235-241.

27. Göttlich, M., Heldmann, M., Göbel, A., Dirk, A.L., Brabant, G., Münte, T.F. (2015). Experimentally induced thyrotoxicosis leads to increased connectivity in temporal lobestructures: a resting state fMRI study. Psychoneuroendocrinology, 56, 100-109.

28. Graham, S., \& Hebert, M. (2011). Writing-to-read: A meta-analysis of the impact of writing and writing instruction on reading. Harvard Educational Review, 81, 710-744.

29. Greenberg, D., \& Ehri, L. C. (2002). Do adult literacy students make the same word-reading and spelling errors as children matched for word-reading age? Scientific Studies of Reading, 6, 221-243.

30. Greenberg, D., \& Lackey, J. (2006). The importance of adult literacy issues in social work practice. Social Work, 51, 177-179.

31. Greenberg, D., Ehri, L. C., \& Perin, D. (1997). Are word-reading processes the same or different in adult literacy students and third-fifth graders matched for reading level? Journal of Education \& Psychology, 89, 262-275.

32. Grigorenko, E. L. (2001). Developmental dyslexia: An update on genes, brains, and environments. Journal of Child Psychology and Psychiatry, 42, 91125.

33. Grosche, M., \& Grünke, M. (2011). Beeinträchtigungen in der phonologischen Informationsverarbeitung bei funktionalen Analphabeten. Z Pädagog Psychol, 25, 277-291.

34. Grotlüschen, A, Buddeberg, K., Dutz, G., Heilmann, L., Stammer, C. (2019). LEO 2018 - Leben mit geringer Literalität. Pressebroschüre. Universität Hamburg. Online unter: http://blogs.epb.uni-hamburg.de/leo. Accessed 30 Apr 2020.

35. Hartwigsen, G., Baumgaertner, A., Price, C. J., Koehnke, M., Ulmer, S., \& Siebner, H. R. (2010). Phonological decisions require both the left and right supramarginal gyri. Proceedings of the National Academy of Sciences of the United States of America, 107, 16494-16499.

36. Hayes, J. R. (1996). A new framework for understanding cognition and affect in writing. In R. Indrisano \& J. R. Squire (Eds.), Perspectives on writing: Research, theory, and practice (pp. 1-27). Newark: International Reading Association.

37. Hebert, M., Kearns, D. M., Hayes, J. B., Bazis, P., \& Cooper, S. (2018 Oct 24). Why children with dyslexia struggle with writing and how to help them. Language, Speech, and Hearing Services in Schools, 49(4), 843-863.

38. Hertz-Pannier, L., Chiron, C., Jambaque, I., Renaux-Kieffer, V., Van de Moortele, P. F., Delalande, O., Fohlen, M., Brunelle, F., \& Le Bihan, D. (2002). Late plasticity for language in a child's non-dominant hemisphere: A preand post-surgery fMRI study. Brain, 125, 361-372.

39. Heth, I., \& Lavidor, M. (2015). Improved reading measures in adults with dyslexia following transcranial direct current stimulation treatment. Neuropsychologia, 70, 107-113.

40. Holloway, V., Gadian, D. G., Vargha-Khadem, F., Porter, D. A., Boyd, S. G., \& Connelly, A. (2000). The reorganization of sensorimotor function in children after hemispherectomy. A functional MRI and somatosensory evoked potential study. Brain, 123, 2432-2444

41. Hornickel, J., Skoe, E., Nicol, T., Zecker, S., \& Kraus, N. (2009). Subcortical differentiation of stop consonants relates to reading and speech-in-noise perception. Proceedings of the National Academy of Sciences of the United States of America, 106, 13022-13027.
42. Horowitz-Kraus, T., DiFrancesco, M., Kay, B., Wang, Y., \& Holland, S. K. (2015a). Increased resting-state functional connectivity of visual- and cognitivecontrol brain networks after training in children with reading difficulties. Neuroimage Clin, 8, 619-630.

43. Horowitz-Kraus, T., Toro-Serey, C., \& DiFrancesco, M. (2015b). Increased resting-state functional connectivity in the Cingulo-Opercular cognitivecontrol network after intervention in children with Reading difficulties. PLOS One, 10, e0133762.

44. Karbe, H., Herholz, K., Halber, M., \& Heiss, W. D. (1998). Collateral inhibition of transcallosal activity facilitates functional brain asymmetry. Journal of Cerebral Blood Flow and Metabolism, 18, 1157-1161.

45. Klostermann, F., Krugel, L. K., \& Ehlen, F. (2013). Functional roles of the thalamus for language capacities. Frontiers in Systems Neuroscience, 7, 32.

46. Koechlin, E., \& Summerfield, C. (2007). An information theoretical approach to prefrontal executive function. Trends in Cognitive Sciences, 11, 229-235.

47. Koyama, M. S., Kelly, C., Shehzad, Z., Penesetti, D., Castellanos, F. X., \& Milham, M. P. (2010). Reading networks at rest. Cerebral Cortex, 20, 25492559

48. Koyama, M. S., Di Martino, A., Kelly, C., Jutagir, D. R., Sunshine, J., Schwartz, S. J., Castellanos, F. X., \& Milham, M. P. (2013). Cortical signatures of dyslexia and remediation: An intrinsic functional connectivity approach. PLoS One, 8 , e55454.

49. Kumar, M., Rana, P., Modi, S., Tyagi, R., Kaur, P., Kanwar, R., Sekhri, T., D'souza, M., \& Khushu, S. (2019). Aberrant intra and inter network resting state functional connectivity in thyrotoxicosis. Journal of Neuroendocrinology, 31, e12683.

50. Küspert, P., \& Schneider, W. (1998). WLLP - Würzburger Leise Leseprobe. Göttingen: Hogrefe.

51. Laird, A. R., Fox, P. M., Eickhoff, S. B., Turner, J. A., Ray, K. L., McKay, D. R., Glahn, D. C., Beckmann, C. F., Smith, S. M., \& Fox, P. T. (2011). Behavioral interpretations of intrinsic connectivity networks. Journal of Cognitive Neuroscience, 23, 4022-4037.

52. Lancaster, J. L., Woldorff, M. G., Parsons, L. M., Liotti, M., Freitas, C. S., Rainey, L., Kochunov, P. V., Nickerson, D., Mikiten, S. A., \& Fox, P. T. (2000). Automated Talairach atlas labels for functional brain mapping. Human Brain Mapping, 10, 120-131.

53. Liu, L., Deng, X., Peng, D., Cao, F., Ding, G., Jin, Z., Zeng, Y., Li, K., Zhu, L., Fan, N., Deng, Y., Bolger, D. J., \& Booth, J. R. (2009). Modality- and task-specific brain regions involved in Chinese lexical processing. Journal of Cognitive Neuroscience, 21, 1473-1487.

54. Liu, X., Gao, Y., Di, Q., Hu, J., Lu, C., Nan, Y., Booth, J. R., \& Liu, L. (2018). Differences between child and adult large-scale functional brain networks for reading tasks. Human Brain Mapping, 39, 662-679.

55. Miller, E. K., \& Cohen, J. D. (2001). An integrative theory of prefrontal cortex function. Annual Review of Neuroscience, 24, 167-202.

56. Morken, F., Helland, T., Hugdahl, K., \& Specht, K. (2017). Reading in dyslexia across literacy development: A longitudinal study of effective connectivity. Neuroimage, 144, 92-100

57. Muller, N. G., \& Knight, R. T. (2006). The functional neuroanatomy of working memory: Contributions of human brain lesion studies. Neuroscience, 139, $51-58$.

58. Müler, R. (2003). Diagnostischer Rechtschreibtest für 1. Klassen (DRT 1) Göttingen: Beltz Test.

59. Nichols, T. E., \& Holmes, A. P. (2002). Nonparametric permutation tests for functional neuroimaging: A primer with examples. Human Brain Mapping, 15, 1-25.

60. O'Reilly, J. X., Beckmann, C. F., Tomassini, V., Ramnani, N., \& JohansenBerg, H. (2010). Distinct and overlapping functional zones in the cerebellum defined by resting state functional connectivity. Cerebral Cortex, 20, 953-965.

61. Patenaude, B., Smith, S. M., Kennedy, D. N., \& Jenkinson, M. (2011). A Bayesian model of shape and appearance for subcortical brain segmentation. Neuroimage, 56, 907-922.

62. Peterson, K. M., Ingvar, M., \& Reis, A. (2009). Language and literacy from a cognitive neuroscience perspective. In D. Olson \& N. Torrance (Eds.), Cambridge handbook of literacy (pp. 152-182). Cambridge: Cambridge University Press.

63. Petrides, M. (2005). Lateral prefrontal cortex: Architectonic and functional organization. Philosophical Transactions of the Royal Society of London. Series B, Biological Sciences, 360, 781-795. 
64. Rüsseler, J., Gerth, I., Boltzmann, M. (2011). Basale Wahrnehmungsfähigkeiten von erwachsenen funktionalen Analphabeten und Analphabetinnen. In: Projektträger im DLR e.V. (ed.) Lernprozesse in Alphabetisierung und Grundbildung Erwachsener: Diagnostik, Vermittlung, Professionalisierung. W. Bertelsmann Verlag, Bielefeld.

65. Rüsseler, J., Menkhaus, K., Aulbert-Siepelmeyer, A., Gerth, I., \& Boltzmann, M. (2012). "AlphaPlus": An innovative training program for reading and writing education of functionally illiterate adults. Creative Education, 3, 357-361.

66. Schurz, M., Wimmer, H., Richlan, F., Ludersdorfer, P., Klackl, J., \& Kronbichler, M. (2015). Resting-state and task-based functional brain connectivity in developmental dyslexia. Cerebral Cortex, 25, 3502-3514

67. Seghier, M. L., \& Price, C. J. (2012). Functional heterogeneity within the default network during semantic processing and speech production. Frontiers in Psychology, 3, 281.

68. Shaywitz, S. E., \& Shaywitz, B. A. (2003). The science of reading and dyslexia. Journal of AAPOS, 7, 158-166.

69. Shaywitz, S. E., \& Shaywitz, B. A. (2008). Paying attention to reading: The neurobiology of reading and dyslexia. Development and Psychopathology, 20, 1329-1349.

70. Silani, G., Lamm, C., Ruff, C. C., \& Singer, T. (2013). Right supramarginal gyrus is crucial to overcome emotional egocentricity bias in social judgments. The Journal of Neuroscience, 33, 15466-15476.

71. Skeide, M. A., Kumar, U., Mishra, R. K., Tripathi, V. N., Guleria, A., Singh, J. P., Eisner, F., \& Huettig, F. (2017). Learning to read alters cortico-subcortical cross-talk in the visual system of illiterates. Science Advances, 3, e1602612.

72. Smith, S. M., Fox, P. T., Miller, K. L., Glahn, D. C., Fox, P. M., Mackay, C. E., Filippini, N., Watkins, K. E., Toro, R., Laird, A. R., \& Beckmann, C. F. (2009). Correspondence of the brain's functional architecture during activation and rest. Proceedings of the National Academy of Sciences of the United States of America, 106, 13040-13045.

73. Smith, S. M., \& Nichols, T. E. (2009). Threshold-free cluster enhancement: Addressing problems of smoothing, threshold dependence and localisation in cluster inference. Neuroimage, 44, 83-98.

74. Spreng, R. N., Mar, R. A., \& Kim, A. S. (2009). The common neural basis of autobiographical memory, prospection, navigation, theory of mind, and the default mode: A quantitative meta-analysis. Journal of Cognitive Neuroscience, 21, 489-510.

75. Spreng, R. N., Stevens, W. D., Chamberlain, J. P., Gilmore, A. W., \& Schacter, D. L. (2010). Default network activity, coupled with the frontoparietal control network, supports goal-directed cognition. Neuroimage, 53, 303-317.

76. Sterzer, P., \& Kleinschmidt, A. (2010). Anterior insula activations in perceptual paradigms: Often observed but barely understood. Brain Structure \& Function, 214, 611-622

77. Tagliazucchi, E., \& Laufs, H. (2014). Decoding wakefulness levels from typical fMRI resting-state data reveals reliable drifts between wakefulness and sleep. Neuron, 82, 695-708

78. Tettamanti, M., Moro, A., Messa, C., Moresco, R. M., Rizzo, G., Carpinelli, A., Matarrese, M., Fazio, F., \& Perani, D. (2005). Basal ganglia and language: Phonology modulates dopaminergic release. Neuroreport, 16, 397-401.

79. Turkeltaub, P. E., Eden, G. F., Jones, K. M., \& Zeffiro, T. A. (2002). Meta-analysis of the functional neuroanatomy of single-word reading: Method and validation. Neuroimage, 16, 765-780

80. Vágvölgyi, R., Coldea, A., Dresler, T., Schrader, J., \& Nuerk, H. C. (2016). A review about functional illiteracy: Definition, cognitive, linguistic, and numerical aspects. Frontiers in Psychology, 7, 1617.

81. Vincent, J. L., Kahn, I., Snyder, A. Z., Raichle, M. E., \& Buckner, R. L. (2008). Evidence for a frontoparietal control system revealed by intrinsic functional connectivity. Journal of Neurophysiology, 100, 3328-3342.

82. Williams, J., Clemens, S., Oleinikova, K., \& Tarvin, K. (2003). The skills for life survey: A national needs and impact survey of literacy, numeracy and ICT skills. London: Dept. for Education and Skills.

83. Wise Younger, J., Tucker-Drob, E., \& Booth, J. R. (2017). Longitudinal changes in reading network connectivity related to skill improvement. Neuroimage, 158, 90-98.

84. Younger, J. W., Randazzo Wagner, M., \& Booth, J. R. (2016). Weighing the cost and benefit of Transcranial direct current stimulation on different Reading subskills. Frontiers in Neuroscience, 10, 262.

85. Žarić, G., Correia, J. M., Fraga González, G., Tijms, J., van der Molen, M. W., Blomert, L., \& Bonte, M. (2017). Altered patterns of directed connectivity within the reading network of dyslexic children and their relation to reading dysfluency. Developmental Cognitive Neuroscience, 23, $1-13$.

86. Zhou, W., Xia, Z., Bi, Y., \& Shu, H. (2015). Altered connectivity of the dorsal and ventral visual regions in dyslexic children: A resting-state fMRI study. Frontiers in Human Neuroscience, 9, 495.

\section{Publisher's Note}

Springer Nature remains neutral with regard to jurisdictional claims in published maps and institutional affiliations.
Ready to submit your research? Choose BMC and benefit from:

- fast, convenient online submission

- thorough peer review by experienced researchers in your field

- rapid publication on acceptance

- support for research data, including large and complex data types

- gold Open Access which fosters wider collaboration and increased citations

- maximum visibility for your research: over $100 \mathrm{M}$ website views per year

At BMC, research is always in progress.

Learn more biomedcentral.com/submissions 